OPEN ACCESS

Edited by:

Dezhi Ning,

Dalian University of Technology, China

Reviewed by:

Wang Chen,

Tsinghua University, China

Hongda Shi,

Ocean University of China, China Yongliang Zhang,

Tsinghua University, China

*Correspondence:

Pengda Cheng

pdcheng@imech.ac.cn

Specialty section:

This article was submitted to

Ocean Solutions,

a section of the journal

Frontiers in Marine Science

Received: 07 March 2021

Accepted: 26 April 2021

Published: 22 June 2021

Citation:

Deng Z, Wang P and Cheng P (2021)

Hydrodynamic Performance of an

Asymmetry OWC Device Mounted on

a Box-Type Breakwater.

Front. Mar. Sci. 8:677030.

doi: 10.3389/fmars.2021.677030

\section{Hydrodynamic Performance of an Asymmetry OWC Device Mounted on a Box-Type Breakwater}

\author{
Zhengzhi Deng ${ }^{1}$, Pinjie Wang ${ }^{1}$ and Pengda Cheng ${ }^{2 *}$ \\ ${ }^{1}$ Ocean College, Zhejiang University, Zhoushan, China, ${ }^{2}$ Institute of Mechanics, Chinese Academy of Sciences, Beijing, China
}

To share the construction and maintenance cost, an asymmetric oscillating water column (OWC) device integrated with a pile-fixed box-typed offshore breakwater is considered experimentally and numerically. A fully nonlinear numerical wave tank is established and validated with the open source solver OpenFOAM. The effects of the width and draft of rear box, and the incident wave height on the wave energy conversion efficiency, reflection and transmission coefficients, and energy dissipation coefficient are examined. In addition, the superiority of the present coupling system, compared to the traditional box-type breakwater, is discussed. With well comparisons, the results show that the existence of the rear breakwater is beneficial for the formation of partial standing waves and further wave energy conversion. In the range of wave heights tested, the higher the incident wave height, the larger the energy absorption efficiency except for the short-wave regimes. Moreover, the OWC-breakwater coupling system can obtain a similar wave blocking ability to the traditional one, and simultaneously extract wave energy and decrease wave reflection.

Keywords: wave energy conversion, oscillating water column, hydrodynamics, experimental and numerical study, energy dissipation

\section{INTRODUCTION}

The growing interest in the conversion and utilization of ocean wave energy source can be traced back to one century ago, since when various forms of wave energy extraction technologies began to appear (Falcão, 2010; You et al., 2010; Qiu et al., 2019). Since the 1980s, the tendency of wave energy exploitation has shifted toward practical, commercial medium-sized devices for the energy supply of remote islands. The oscillating water column (OWC) wave energy converter (WEC) is considered to be one of the most widely used technologies for its simple structure, easy installation and maintenance, and non-corrosive mechanical components (Falcão, 2010; Heath, 2012), whose main working principle is that the air-chamber pressure fluctuation induced by the heave motion of interior water column feeds the generator mounted on the top to fulfill the energy conversion.

At an early stage, the study on OWC device is concentrated on the hydrodynamic performance and efficiency optimization in terms of the structural configuration of a stand-alone OWC device. Evans (1976) employed the ship-hydrodynamic theory to theoretically investigate the hydrodynamic properties of an OWC device by simplifying the internal water surface as a weightless piston. Later, Evans (1982) put forward the surface air pressure distribution theory and derived the expression of the optimal energy conversion efficiency of OWC devices. In order to further improve the hydrodynamic performance, in the literature, many scholars attempted 
to optimize the structural configuration of a single-air-chamber OWC device. Count and Evans (1984) suggested extending the side wall along the direction of wave incidence to expand the structure of air chamber and strengthen the wave-focusing effect. Deng et al. (2013) employed the matched eigenfunction expansion method to study the structure optimization of a coaxial structure-supported OWC device, and presented the optimal bottom opening in the range of $\pi / 2-5 \pi / 4$. Extending the work by Deng et al. (2013) and Deng et al. (2014) further established a theoretical OWC model with a V-shaped channel at the bottom. They concluded that the existence of the V-shaped channel can significantly increase the conversion efficiency and widen the range of highly effective wave frequencies. Ashlin et al. (2016) focused on the configuration of the OWC bottom profile and presented that the device with a circular curve bottom profile has better performance. Vyzikas et al. (2017) compared the capability of OWC and U-OWC devices experimentally, and proposed preliminary opinions on how to optimize the structural form of traditional OWC devices. Ning et al. (2020a) carried out a numerical study on the influence of the thickness of the front wall of onshore U-OWC device, and the results indicated that when the thickness of the front wall increases, the efficiency of the OWC device will increase. Furthermore, Ning et al. (2020b) added a circle side wall to the outside of the single chamber OWC device, forming a structure of a dual chamber. The hydrodynamic performance of the cylindrical dual-chamber OWC device was experimentally and numerically investigated, and it was found that the efficiency of dual-chamber device was better than that of a single-chamber device.

To share the construction and maintenance cost, coupling OWC devices into other marine structures, such as breakwaters, is proposed and received much attention (Mustapa et al., 2017; Zhao et al., 2019; Zheng et al., 2019). Martins-Rivas and Mei (2009a,b) theoretically investigated the hydrodynamic performance and optimum absorption efficiency of an isolated OWC device mounted on a breakwater and a straight coast. Further, Lovas et al. (2010) considered an OWC device installed at a coastal corner under the framework of linear water wave theory. He et al. (2013) proposed a multi-functional floating rectangular breakwater mounted by two asymmetric OWC devices on both sides. They experimentally investigated the hydrodynamic performance and concluded that the integration is a viable option for cost-sharing between wave energy capturing devices and shore-protection structures, and thus enhance the cost-effectiveness of wave energy utilization. From an experimental and numerical point of view, Howe and Nader (2017) compared and discussed the hydrodynamic performances between the single OWC device and the OWC-breakwater coupling device, and discovered that the highly effective wavefrequency bandwidth of the coupling system is more than twice as the single one. On the basis of fully non-linear HOBEM model, Ning et al. (2019) predicted the hydrodynamic response of an OWC device over a stepped bottom, and the optimization scheme of step geometry and position was summarized. Deng et al. (2020) employed the open source platform OpenFOAM to deeply analyze the hydrodynamic performance of the offshore OWC device constructed on a rectangular underwater breakwater, and proposed an optimum structural configuration that can convert more wave energy over a wider wave-frequency range. Integrating the OWC device with a vertical tubular structure, Zheng et al. (2020) employed the matched eigenfunction expansion method to solve the problems of wave scattering and radiation under finite water depth. They found that a thinner chamber wall thickness offers benefits to wave power extraction in terms of a broader primary band of power capture factor response.

Most of the previous studies on the coupling systems of OWC and breakwater have focused on optimizing the structural shape of the OWC device, such as setting a U-type channel and a Vtype opening, or finding the superior geometric dimensions of the OWC device, especially for the chamber width and opening ratio. The main concern of these studies is still the pneumatic efficiency of the OWC device, neglecting the wave dissipation and wave-blocking performance of the breakwater. When the size of the breakwater changes, it is challenging to maintain the overall hydrodynamic performance of the integrated system to an excellent level, that is, it can not only improve the wave energy extraction efficiency of the OWC device but also enable the breakwater to eliminate wave remarkably to create satisfactory navigation conditions.

In this paper, in order to block the wave transmission and simultaneously decrease wave reflection to improve the navigation safety of ships, an isolated OWC device equipped on the waveward side of a pile-fixed box-type breakwater is considered. Using the hybrid approach of experiments and numerical simulations, the effects of the structural parameters of the breakwater, such as the immersion depth, extended width, on the hydrodynamic performance (i.e., energy conversion coefficient, transmission, and reflection coefficients) are examined. In addition, the wave nonlinearity (wave height) is discussed. The article is structured as follows. The setup and information of experiment are presented in section 2. The computational principle and numerical method in OpenFOAM and wave generation toolbox waves2Foam are described in section 3 . The verification and validation of the numerical model is discussed in section 4. Finally, the conclusions are drawn in section 5 .

\section{EXPERIMENT}

\subsection{Experimental Setup}

The small-scale experiments were carried out in the wave flume at the Ocean Test Hall of Zhejiang University, China. The wave flume is $35 \mathrm{~m}$ long, $0.6 \mathrm{~m}$ wide, and its maximum water depth is $0.8 \mathrm{~m}$. One end of the flume is fitted with a $1.5 \mathrm{~m}$ long pistontype wave-maker capable of generating both regular and irregular waves. At the other end of the flume, a $3 \mathrm{~m}$ long artificial beach is installed to absorb the outgoing wave. The OWC physical model is located in the middle of the flume and its front wall is 15.5 $\mathrm{m}$ away from the wave-maker. The width of the OWC model is equal to the flume in order to prevent wave diffraction, so that the experiments can be regarded as two-dimensional. The OWC physical model is made up of transparent Perspex sheets with 


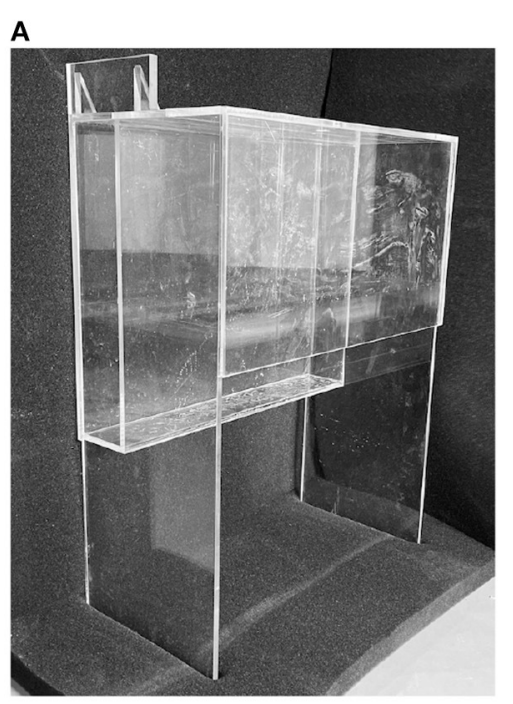

Physical diagram

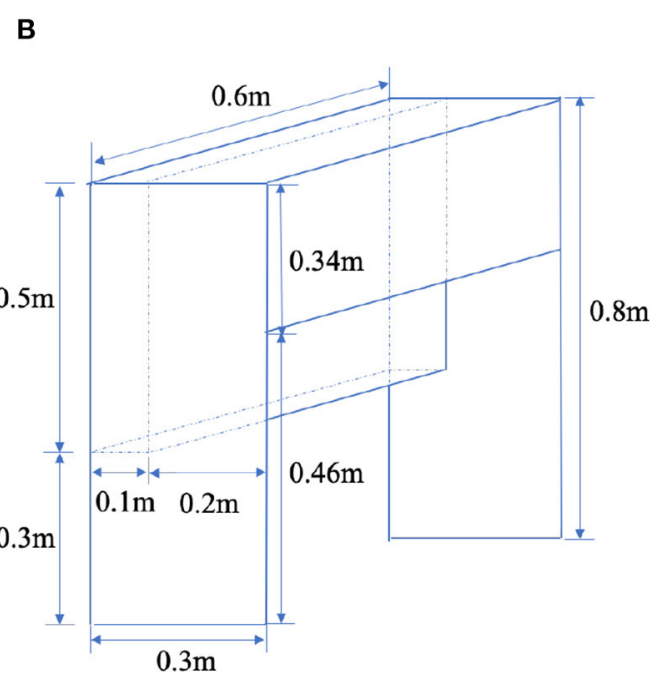

Geometric sketch

FIGURE 1 | Schematic of the oscillating water column (OWC) model. (A) Physical diagram (B) Geometric sketch.

thickness of $10 \mathrm{~mm}$, as shown in Figure 1A, and the geometric sketch is illustrated in Figure 1B.

Figure 2 shows the experimental layout, including the locations of seven capacitance-type wave gauges $\left(G_{1}-G_{7}\right)$ and two pressure transducers $\left(S_{1}, S_{2}\right)$. The nearest gauge to the wave maker, $G_{1}$, is to monitor the generated incident wave. The time series of surface elevations monitored by $G_{2}$ to $G_{4}$ is used to separate the incident and reflected waves based on the two-point method (Goda and Suzuki, 1976). $G_{5}$ and $G_{6}$ are used to record the free surface elevations inside the chamber. $G_{7}$ is placed behind the structure to evaluate the transmission coefficient. In addition, two pressure transducers $S_{1}$ and $S_{2}$ are arranged below the orifice to monitor the relative pressure drop between the inside and outside of the chamber. In all tests, the data are collected by DJ800 data acquisition instrument of Tianjin Hydro-Technical Institute with $100 \mathrm{~Hz}$ sampling rate and $40 \mathrm{~s}$ duration time. In order to reduce the experimental uncertainty, experiments under each wave condition are repeated at least three times and the averaged value is taken as the final result. It is noted that, in this paper, to avoid the effect of multi-reflection, the stable data segment is selected in data processing.

\subsection{Physical Model and Test Wave Conditions}

As sketched in Figure 1, unlike the traditional single OWC device, the proposed device is integrated with a box-like structure, serving as a pile-fixed offshore breakwater. The front plate with a constant small submerged depth is considered, i.e., $d_{1}=0.04 \mathrm{~m}$, which is beneficial for the wave energy entering the chamber and being absorbed. To model the energy extracting process of the power-takeoff (PTO) system, a long and narrow slot in the middle of the chamber ceiling was made with an opening rate of $1 \%$ (divided by the cross-sectional area of the chamber). The air chamber width, $a$, is fixed at $0.2 \mathrm{~m}$. Besides, $B$ and $d_{2}$ denotes the width and draft of the rear box, respectively. In experiments, two widths of the rear box $(B=0.1 \mathrm{~m}$ and 0.3 $\mathrm{m}$ ) were tested where the $d_{2}$ remained $0.2 \mathrm{~m}$.

On the basis of Froude similarity criterion, a length scaling of 1:25 was chosen, and the experimental wave conditions and model dimensions tested here are listed in Table 1. The water depth is fixed at $0.5 \mathrm{~m}$, and two incident wave heights are selected $\left(H_{i}=0.03 \mathrm{~m}\right.$ and $\left.0.05 \mathrm{~m}\right)$. The period of regular incident wave, $T$, varies from $1 \mathrm{~s}$ to $2.4 \mathrm{~s}$ with an increment of $0.2 \mathrm{~s}$, and the corresponding wave length, $\lambda$, changes from $1.5 \mathrm{~m}$ to $5.0 \mathrm{~m}$. The range of dimensionless parameter $k h$ obtained by wave number is 0.6 to 2.1 .

It is worth mentioning that because the scale of the physical model is small, the effect of air compressibility is neglected in this paper (Xu et al., 2016).

\subsection{Hydrodynamic Characteristics Calculation}

Generally speaking, the time-averaged hydrodynamic energy extracted by the OWC wave energy conversion device over a wave period, $E_{O W C}$, can be expressed as Equation (1):

$$
E_{O W C}=\frac{a w}{T} \int_{t_{0}}^{t_{0}+T} P_{a}(t) \dot{\eta}(t) d t
$$

where $a$ denotes for chamber width, $P_{a}$ denotes for the pneumatic pressure, $\eta$ is the vertical displacement of the water surface inside the chamber, $T$ is the incident wave period, $t_{0}$ is a reference time instance, and $w=1$ for the two-dimensional model. 


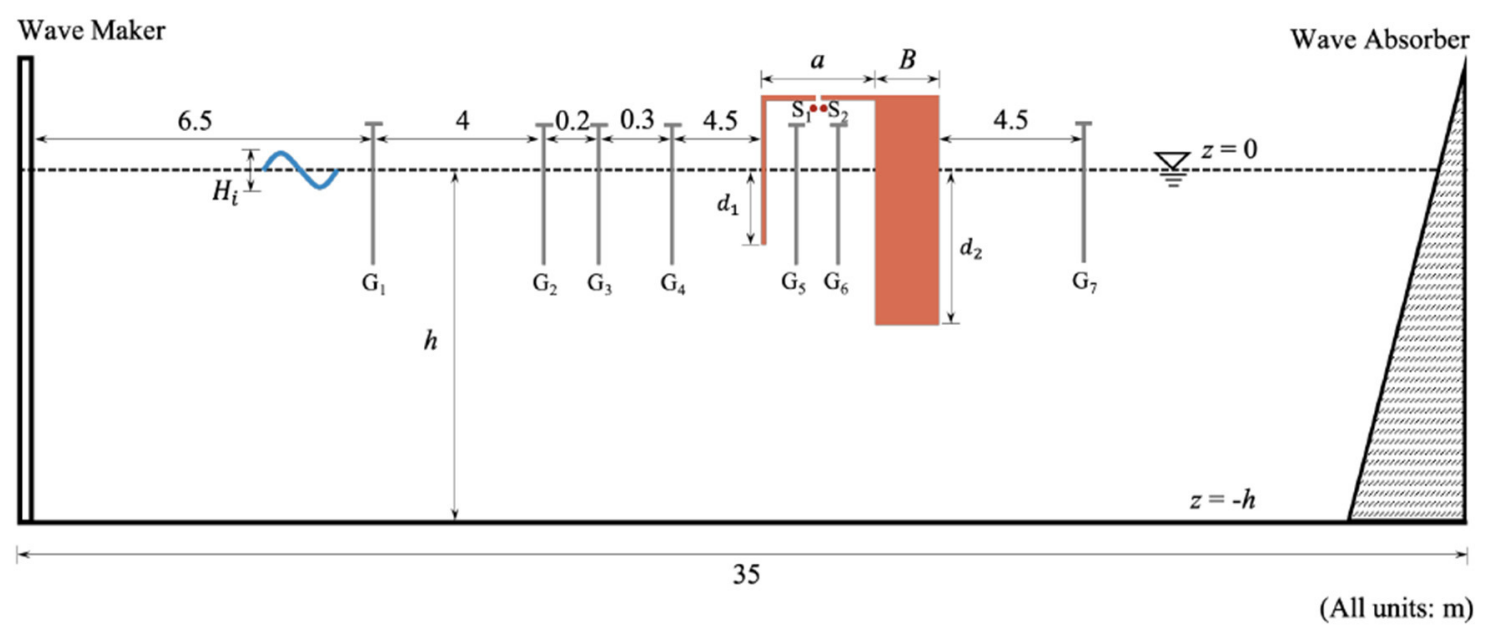

FIGURE 2 | Experiment layout.

The incident wave power here is defined as Equation (2):

$$
P_{i n c}=\frac{\rho g A_{i}^{2} \omega}{4 k}\left(1+\frac{2 k h}{\sinh 2 k h}\right),
$$

where $\rho$ is the water density, $A_{i}$ is the incident wave amplitude, $\omega$ denotes the angular velocity, $k$ represents the wave number, and $g$ is the gravity acceleration.

The wave energy conversion efficiency, $\xi$, is calculated by Equation (3):

$$
\xi=\frac{E_{O W C}}{P_{i n c} \cdot w} .
$$

By using the two-point method to separate the reflected wave from the time series monitored by wave gauges $G_{2}$ to $G_{4}$, the reflection coefficient, $C_{r}$, can be computed by Equation (4):

$$
C_{r}=\frac{H_{r}}{H_{i}},
$$

where $H_{r}$ represents the reflected wave height and $H_{i}$ represents the incident wave height.

After the wave energy is partially reflected and extracted by the OWC device, the rest transmits underneath the structure and is measured by $G_{7}$. Similarly, the transmission coefficient, $C_{t}$, can be calculated by

$$
C_{t}=\frac{H_{t}}{H_{i}}
$$

where $H_{t}$ represents the transmitted wave height.

In order to quantify the energy loss due to vortex shedding and fluid separation, the energy dissipation ratio, $C_{d}$, is defined by,

$$
C_{d}=1-C_{r}^{2}-C_{t}^{2}-\xi \text {. }
$$

TABLE 1 | Experimental test conditions and model size.

\begin{tabular}{lccccccccc}
\hline Parameters & Value & & & & & & & \\
\hline Water depth $h(\mathrm{~m})$ & 0.5 & & & & & & & \\
Wave height $H_{i}(\mathrm{~m})$ & 0.03 & 0.05 & & & & & & \\
Wave period $T(\mathrm{~s})$ & 1.0 & 1.2 & 1.4 & 1.6 & 1.8 & 2.0 & 2.2 & 2.4 \\
Wavelength $\lambda(\mathrm{m})$ & 1.51 & 2.05 & 2.57 & 3.08 & 3.57 & 4.06 & 4.53 & 5.00 \\
$k h$ & 2.08 & 1.53 & 1.22 & 1.02 & 0.88 & 0.77 & 0.69 & 0.63 \\
Draft $d_{1}(\mathrm{~m})$ & 0.04 & & & & & & & \\
Draft $d_{2}(\mathrm{~m})$ & 0.2 & & & & & & & \\
Chamber width $a(\mathrm{~m})$ & 0.2 & & & & & & & \\
Rear box width $B(\mathrm{~m})$ & 0.1 & 0.3 & & & & & & \\
& & & & & & & & & \\
\hline
\end{tabular}

\section{NUMERICAL MODEL}

\subsection{Governing Equations}

To complement the experimental scenarios and obtain more detailed flow information, a nonlinear numerical wave tank (NWT) is developed employing the open-source software package OpenFOAM, and the sketch of NWT is plotted in Figure 3. The air inside the chamber is considered as incompressible for small-scale physical model. In order to describe the incompressible two-phase flow motion, the toolbox waves2Foam, programmed based on the interFoam solver, is used to solve the Reynold averaged Navier-Stokes equations, including the continuity (Equation (7)) and momentum conservation equation (Equation (8)). Moreover, the Volume of Fluid (VOF) technique (Hirt and Nichols, 1981) is employed to capture the air-water interface. In Cartesian coordinate system, the basic governing equations are written as follows:

$$
\nabla \cdot \vec{U}=0
$$




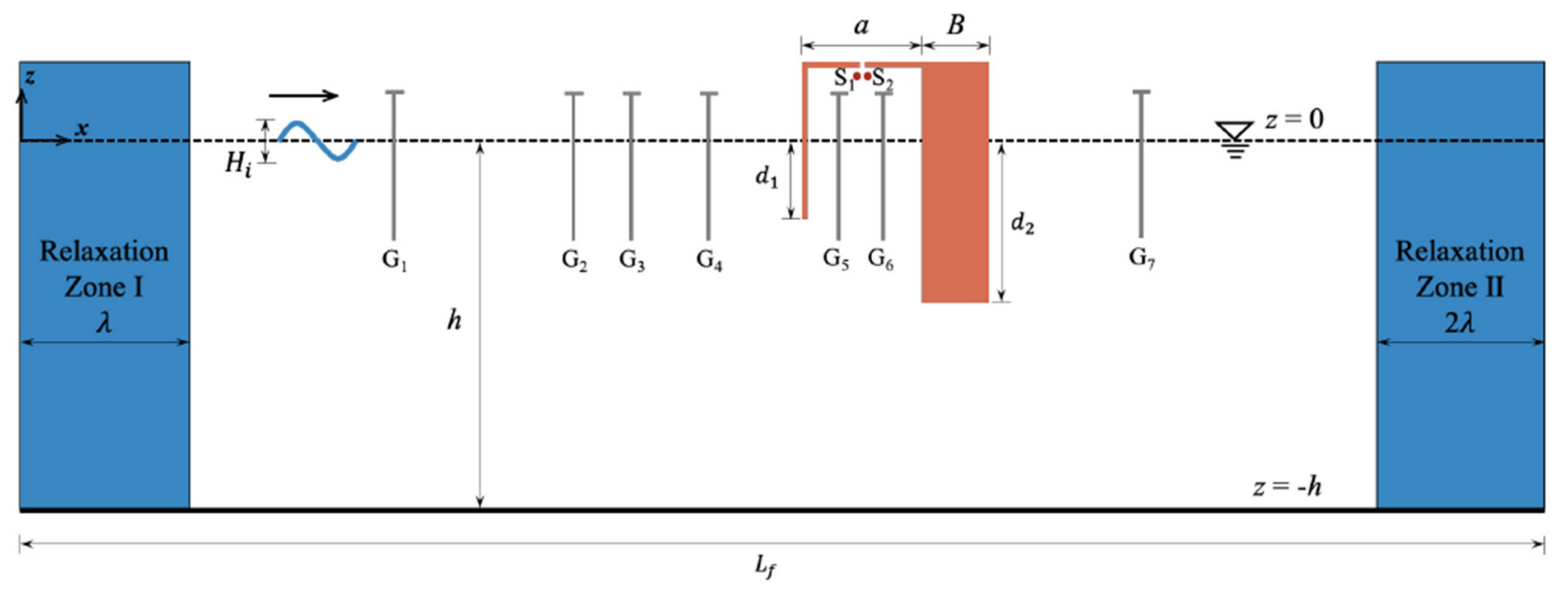

FIGURE 3 | Sketch of numerical wave tank.

$$
\begin{array}{r}
\frac{\partial \rho \vec{U}}{\partial t}+\nabla \cdot(\rho \vec{U} \vec{U})-\nabla \cdot\left(\mu_{e f f} \nabla \cdot \vec{U}\right) \\
=-\nabla p^{*}-\vec{g} \vec{X} \cdot \nabla \rho+\nabla \vec{U} \cdot \nabla \mu+\sigma \kappa \nabla \alpha .
\end{array}
$$

Here, $\vec{U}$ is the velocity vector, $\rho$ is the fluid density, $p^{*}$ is the pseudodynamic pressure, $\vec{g}$ is the acceleration of gravity, $\vec{X}$ is the position vector, $\mu_{\text {eff }}$ is the efficient dynamic viscosity, $\mu$ is the dynamic viscosity of water or air, $\sigma$ is the surface tension coefficient, and $\kappa$ is the curvature of the interface. It is worth mentioning that $\mathrm{k}-\omega$ SST buoyancy model (Devolder et al., 2018) is adopted to describe the turbulence phenomenon.

For the VOF technique, a single-phase function $(\alpha)$ defined as the proportion of water volume in each cell is introduced, and the phase transport can be described by the following convection equation:

$$
\frac{\partial \alpha}{\partial t}+\nabla \cdot(\vec{U} \alpha)=0 .
$$

Considering the realistic meaning of phase function, it is essential to apply the MULES (multidimensional universal limiter for explicit solution) (Deshpande et al., 2012) solver to guarantee $\alpha$ being between 0 and 1 , where $\alpha=0$ denotes gas phase and $\alpha=1$ represents liquid phase. Additionally, to ensure clarity of the free surface between gas and liquid phase, numerical dissipation should be minimized as much as possible. Weller (2002) introduced an artificial compression term to keep the interface sharp and the revised format is as follows:

$$
\frac{\partial \alpha}{\partial t}+\nabla \cdot(\vec{U} \alpha)+\nabla \cdot U_{r} \alpha(1-\alpha)=0,
$$

where $U_{r}$ is the compression velocity between phase water and phase air working only at interface (Rusche, 2003).

Then, $\rho$ and $\mu$ are computed by weighted calculation of air and water on the basis of phase function:

$$
\rho=\alpha \rho_{\text {water }}+(1-\alpha) \rho_{\text {air }},
$$

$$
\mu=\alpha \mu_{\text {water }}+(1-\alpha) \mu_{\text {air }}
$$

\subsection{Boundary Conditions}

To solve the governing equations uniquely, it is essential to specify the reasonable boundary conditions in the numerical wave tank. OpenFOAM provides many classical boundary conditions, among which the no-slip condition is applied for the rigid structure walls and seabed, and the pressure outlet boundary condition is employed on the atmosphere boundary. As for the wave generation and absorption, the toolbox waves2Foam is utilized to setup the special boundary conditions. The secondorder Stokes wave is used in this study to simulate the regular progressive waves. The free surface elevation and velocity components at the inlet boundary can be acquired theoretically as follows:

$$
\begin{array}{r}
\eta=-\frac{k A^{2}}{2 \sinh 2 k h}+A \cos (k x-\omega t) \\
+\frac{A^{2} k}{4} \frac{\cosh k h(\cosh 2 k h+2)}{\sinh ^{3} k h} \cos 2(k x-\omega t), \\
\left\{\begin{array}{l}
u=A \omega\left[\frac{\cosh k(z+h)}{\sinh k h} \cos (k x-\omega t)\right. \\
\left.+\frac{3}{4} A k \frac{\cosh 2 k(z+h)}{\sinh ^{4} h h} \cos 2(k x-\omega t)\right], \\
w=A \omega\left[\frac{\sinh k(z+h)}{\sinh k h} \sin (k x-\omega t)\right. \\
\left.+\frac{3}{4} A k \frac{\sinh 2 k(z+h)}{\sinh ^{4} k h} \sin 2(k x-\omega t)\right],
\end{array}\right.
\end{array}
$$

where $\eta$ is the free surface elevation, $u$ is the horizontal velocity of water, $w$ is the vertical velocity of water, $A$ is the wave amplitude, $\omega$ is the wave angular frequency, $k$ is the wave number, $z$ is the vertical distance from the still water level, and $h$ is the water depth.

In order to eliminate the influences of the reflected/multireflected waves, two relaxation zones are deployed at both ends 
of the NWT. Any physical quantity in the relaxation zones can be calculated by the following equation:

$$
\beta=\alpha_{R} \beta_{\text {computed }}+\left(1-\alpha_{R}\right) \beta_{\text {target }}
$$

where $\alpha_{R}$ is a weighting factor dependent on the location in the relaxation zone and $\beta$ is the aimed variable (Jacobsen et al., 2012), which is the weighted combination of the RANS solution in the domain and the theoretical solution based on the boundary definition.

\subsection{Numerical Method}

The finite volume method (FVM) is used to deal with the governing equations and the computational domain is discretized into a series of non-repeated control volumes, namely cells. Each cell is represented by a node, which locates in the center and the flow field information is stored here.

PIMPLE algorithm is performed to settle the pressurevelocity coupling problem in the incompressible N-S equations, which comprises two algorithms, i.e., Pressure Implicit with Splitting of Operator (PISO) and Semi-Implicit Method for Pressure Linked Equation (SIMPLE), and is mainly inherited from the former algorithm.

As for the numerical discretization schemes in this paper, the Euler scheme is utilized for the time integration, the Gauss limitedLinearV1 scheme for the advective term and the Gauss linear corrected scheme for the Laplacian terms.

\section{VERIFICATION AND VALIDATION}

\subsection{Numerical Convergence Study}

On the basis of FVM, the first step of the numerical model verification is to confirm that the grid setting of the empty wave tank has reached the convergence state, indicating that the calculation has both excellent accuracy and high speed. Five mesh numbers were selected for testing in the range of unit wavelength $\left(N_{x}\right)$ and unit wave height $\left(N_{z}\right)$. It can be concluded that when $N_{x}=100$ and $N_{z}=12$, the wave generated by waves 2 Foam is capable of maintaining a stable height along the propagation and the calculation cost is relatively low, which is similar to previous studies (Hu et al., 2016). For the time step, referring to the works by Deng et al. (2019), $N_{t}=1,000$ (discretized number per wave period) is chosen.

In addition, after integrating the OWC model, the flow fields (vortex shedding) near the structure and at the orifice are more complex. Therefore, further refined mesh scheme is needed. To examine the sensitivity of the grid around the structure, three grid discretization schemes including OWC model are considered, i.e., coarse, medium, and fine. The internal water surface elevation and pressure drop are plotted in Figure 4. Despite the slight deviation at the peaks, the overall results under all discrete schemes are quite consistent with each other. Considering both the numerical accuracy and time cost, the medium mesh scheme with the first grid height of $3 \mathrm{~mm}$ near the structure boundaries and at least 4 grids at the orifice is chosen.

\subsection{Comparison Between Experimental and Numerical Results}

After the convergency test, the well-validated numerical model is adopted to simulate the interaction between water wave and OWC device. The sketch of the NWT is displayed in Figure 3 and the setup is the same as the experimental one. Moreover, the lengths of relaxation zone I and II are 1.5 and 2 times of incident wavelength, respectively.

The comparison among experiment and numerical simulation for the internal water surface displacement and relative air pressure drop is demonstrated in Figure 5. The results for $B=1.5 a, d_{2}=0.2 \mathrm{~m}, H_{i}=0.03 \mathrm{~m}$, and $T=2.4 \mathrm{~s}$ are considered. In general, except for the discrepancies in the peak and trough, the results for experiment and simulation can meet good consistence. The slight discrepancies may be due to the nonlinear effects caused by the different methods of wave generation between experiments and numerical simulations, since different high-order wave components has different ability entering the inside of water column, which directly affects the hydrodynamic performance at peaks and troughs.

Moreover, the comparisons on the hydrodynamic performance between experiments and numerical simulations, including the wave energy conversion efficiency $(\xi)$, reflection $\left(C_{r}\right)$ and transmission $\left(C_{t}\right)$ coefficients, and energy dissipation coefficient $\left(C_{d}\right)$, are also carried out. The results for one incident wave height $\left(H_{i}=0.03 \mathrm{~m}\right)$ together with two box widths ( $B=0.5 a$ and $1.5 a)$ are illustrated in Figure 6, where $d_{2}$ is fixed to $0.2 \mathrm{~m}$. It can be found that $\xi, C_{r}$ and $C_{t}$ of the numerical prediction are basically consistent with the experimental ones. As for the energy dissipation coefficient $C_{d}$, the experimental results are slightly larger than the numerical ones, which may be attributed to the fact that the selected turbulence closure model in simulations cannot sufficiently duplicate the turbulent processes. Besides, the energy dissipation coefficient is not directly observed, but is approximately calculated by Equation (6) that amplifies the error of reflection and transmission coefficients. Overall, it is proved from the comparisons that the numerical model employed here has high accuracy and credibility in predicting the hydrodynamic properties of the OWC device.

\section{RESULTS AND DISCUSSION}

In this section, the numerical simulation of the OWC device mounted on a box-type breakwater, employing the well-validated NWT model, was carried out to explore the hydrodynamic performance, which is affected by the width and draft of the rear box, as well as the incident wave height. For the spatial discretization of the computational domain, the grid discretization strategy described in section 4.1 is adopted. Time step is set as $T / 1,000$. It is noted that the effects of the draft of front wall and chamber size on the hydrodynamic performance have received much attention in the literature; in this paper, the focus will be mainly paid to the structure configuration of the box. 


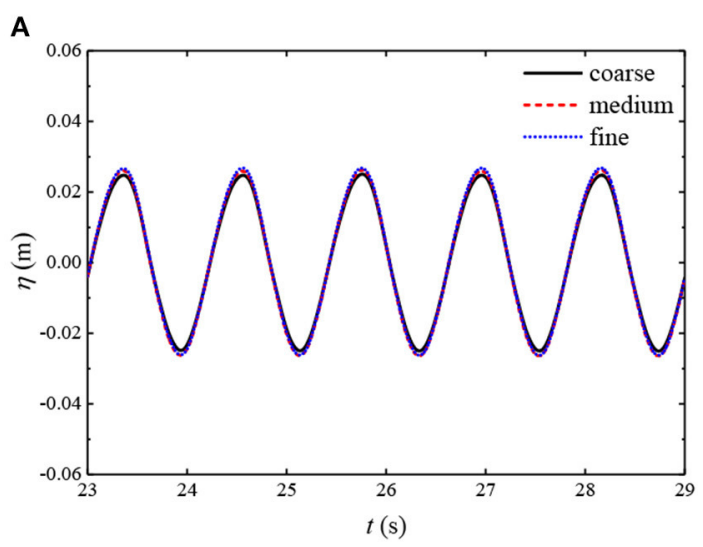

Internal water surface elevation

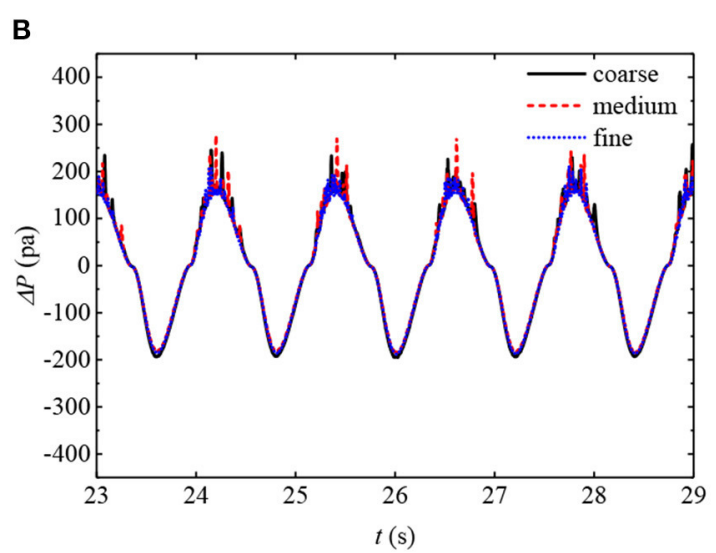

Relative pressure drop

FIGURE 4 | Mesh convergence around the oscillating water column (OWC) device. (A) Internal water surface elevation. (B) Relative pressure drop.

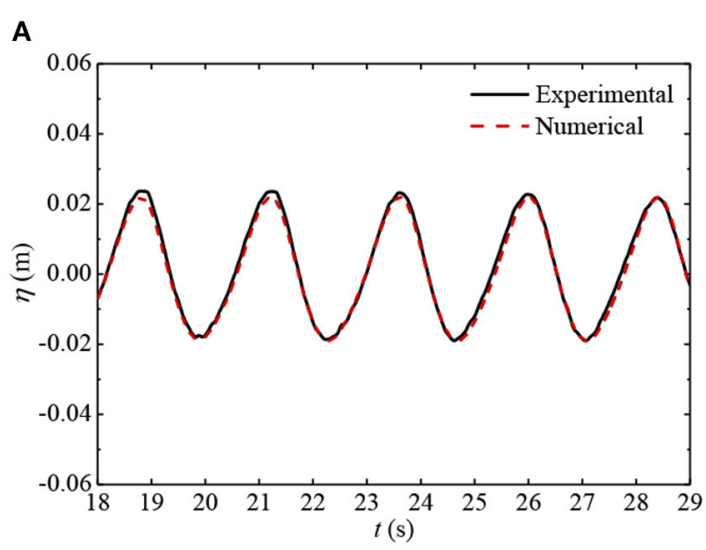

Internal surface displacement

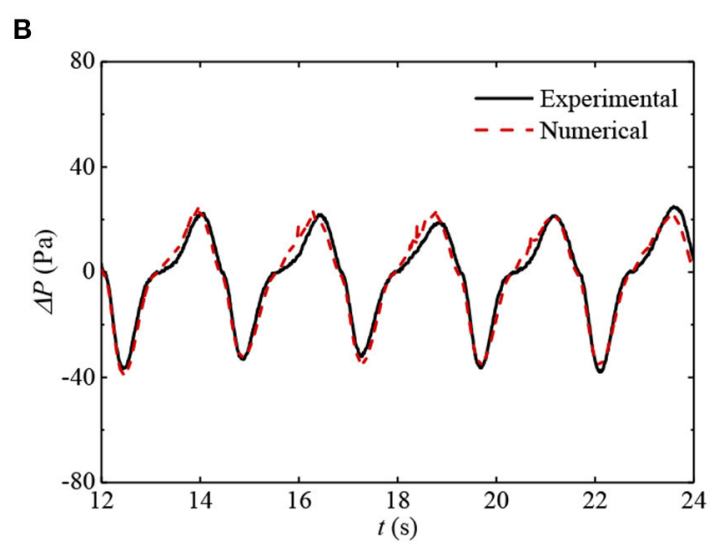

Relative pressure drop

FIGURE 5 | Time series of internal surface displacements and relative pressure drops. In all cases, $B=1.5 a, d_{2}=0.2 \mathrm{~m}, H_{i}=0.03 \mathrm{~m}$, and $T=2.4 \mathrm{~s}$. (A) Internal surface displacement. (B) Relative pressure drop.

\subsection{Effects of the Width of Rear Box}

In this section, the effects of the width of rear box were studied. The draft of rear box is fixed at $d_{2}=0.2 \mathrm{~m}$, and the incident wave height $H_{i}$ is $0.05 \mathrm{~m}$. The $\mathrm{OWC}$ devices equipped with six rear box widths, ranging from $B=0.05 a$ to $2.5 a$, were examined in terms of hydrodynamic coefficients $\xi, C_{r}, C_{t}$, and $C_{d}$.

Figure 7 indicates that the four hydrodynamic parameters vary against the non-dimensional wave frequency $k h$ for different rear-box widths, and the curves are all fitted based on B-Spline algorithm. Overall, all coefficients are unidirectional with the change of rear box width. As the width of rear box increases, the energy conversion efficiency of OWC device is improved over the full frequency domain considered and the energy dissipation decreases, as shown in Figures 7A,D. Meanwhile, more waves are reflected, especially in the long-wave regimes, and correspondingly less waves transmit across the OWC system, as depicted in Figures 7B,C. It is worth noting that $B=0.05 a$ means the width of rear box is the same as the thickness of front-plate, that is, there is no breakwater platform, while $B=2.5 a$ means the rear box width equaling to the water depth. The maximum of the wave energy conversion efficiency of the former OWC device in Figure 7A is 0.63 , while that of the latter one is 0.75 , increasing about $19 \%$. If taking $\xi=0.6$ as the high-efficient benchmark, the bandwidth of high performance for the OWC device with widest rear box is $1.24 \leq k h \leq 2.08$, which is as about six times as the one with thinnest box. As for the reflection coefficient $C_{r}$ in Figure 7B and transmission coefficient $C_{t}$ in Figure 7C, the maximum increase in $C_{r}$ and the maximum decrease in $C_{t}$ of the OWC device equipped with the widest box are 356 and 


\section{A}

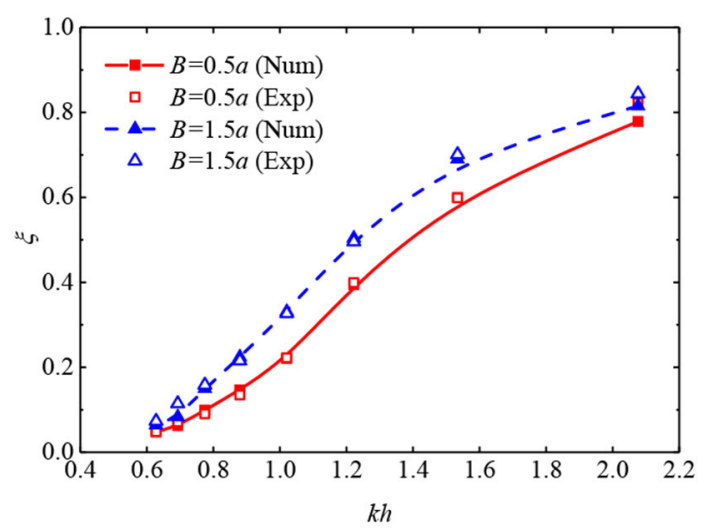

Energy conversion efficiency

C

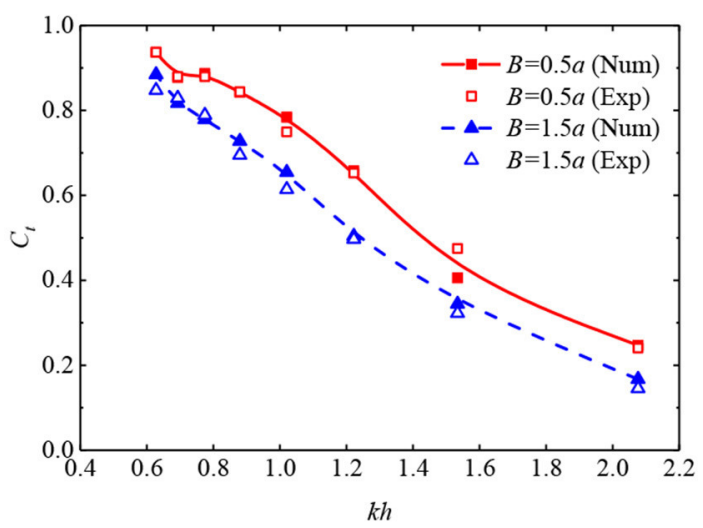

Transmission coefficient
B

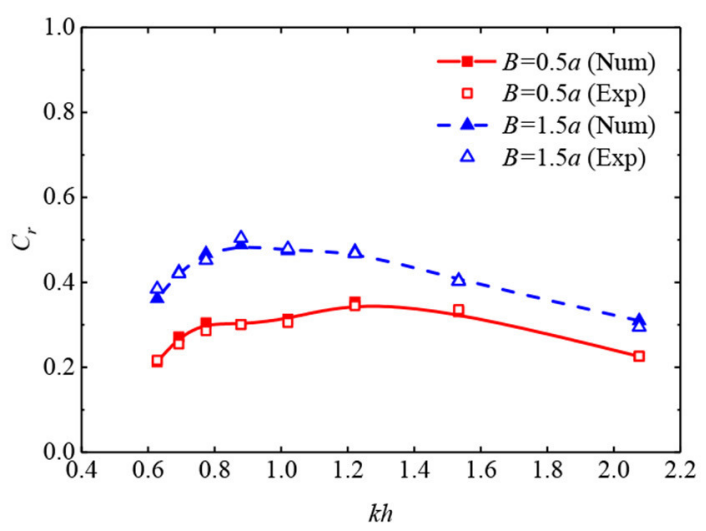

Reflection coefficient

D

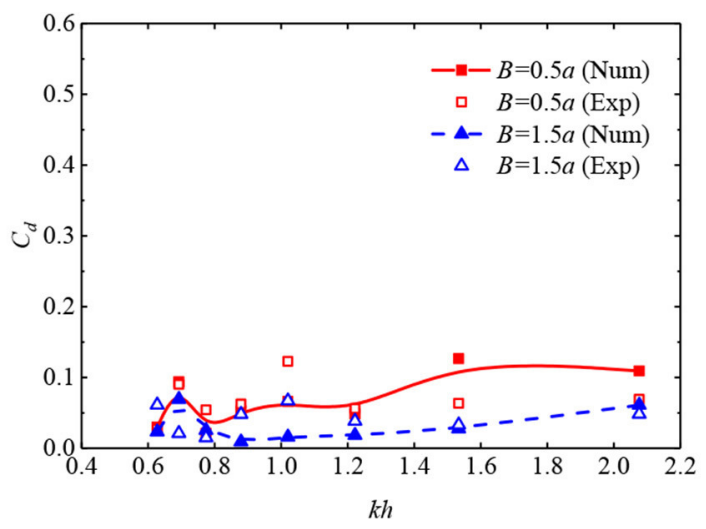

Energy dissipation coefficient

FIGURE 6 | Comparisons of the experimental and numerical results. In all cases, $a=0.2 \mathrm{~m}, H_{i}=0.03 \mathrm{~m}$, and $d_{2}=0.2 \mathrm{~m}$. (A) Energy conversion efficiency. (B) Reflection coefficient. (C) Transmission coefficient. (D) Energy dissipation coefficient.

$59 \%$, respectively, compared to the OWC device without the breakwater platform.

The energy dissipation coefficient $C_{d}$ is presented in Figure 7D. When the dimensionless frequency $k h$ is 0.69 , there seems to be a peak in the fitting curve. This is the fact that at this frequency, the energy extraction efficiency and reflection coefficient of the wave energy device change very little, but the decrease of transmission coefficient is relatively obvious. Therefore, when calculated according to Equation (6), the dissipation ratio has a small increase. It is worth noting that the ordinate range of the dissipation ratio is 0 to 0.4 , not 0 to 1 as in other graphics, so the peak phenomenon is not obvious in value.

The above results show that the OWC device coupled with a relative wider breakwater platform can not only greatly improve the wave-blocking ability but also be beneficial for the wave energy extraction by the OWC device. However, it should be noted that from the engineering application perspective, infinite wide breakwater platform is unrealistic, which should be determined depending on the local engineering requirements. In the present work, the maximum width of $B=h$ (i.e., $B=2.5 a$ ) is considered.

\subsection{Effects of the Draft of Rear Box}

This section attempts to explore the effects of the draft of rear box on the hydrodynamic performance of OWC device. Based on the above investigations, the rear box width of $B=2.5 a$ is chosen, and the incident wave height $H_{i}$ is still $0.05 \mathrm{~m}$. Four draft schemes were selected, ranging from $d_{2}=d_{1}$ to $10 d_{1}$.

Figure 8 illustrates the variations of hydrodynamic properties against dimensionless coefficient $k h$. As expected, increasing the immersion depth of the rear box is beneficial for the formation of partial standing waves due to the blocking effect of the rear wall. As a result, the energy conversion efficiency is improved over a wider wave-frequency range. However, if further increasing 


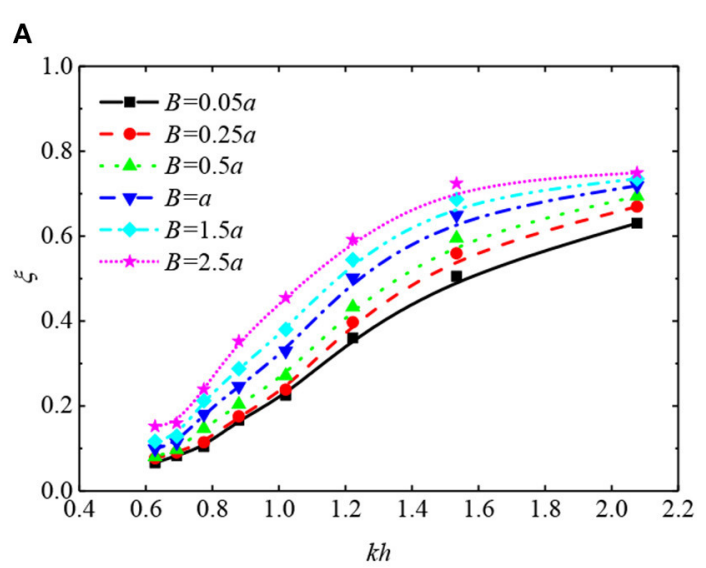

Energy conversion efficiency

C

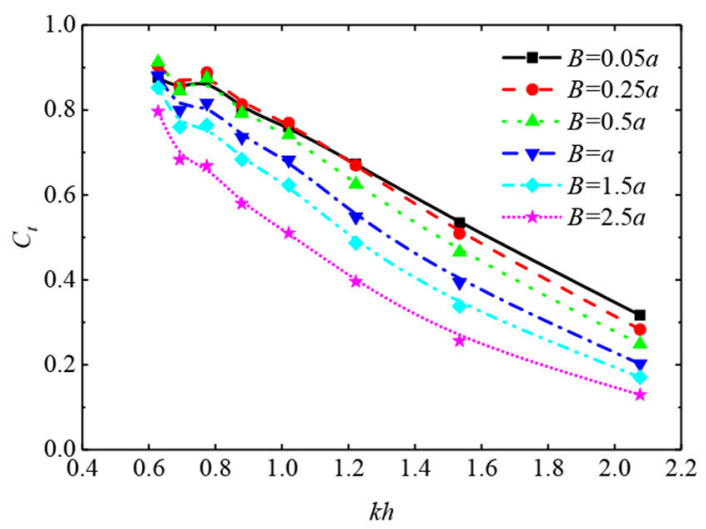

Transmission coefficient
B

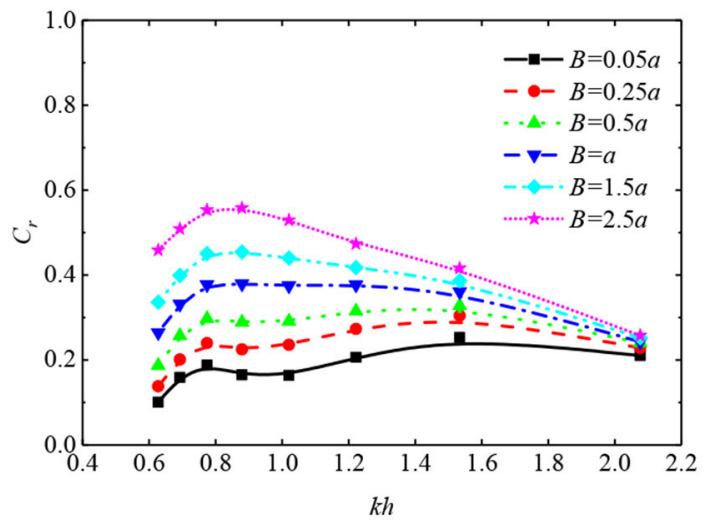

Reflection coefficient

D

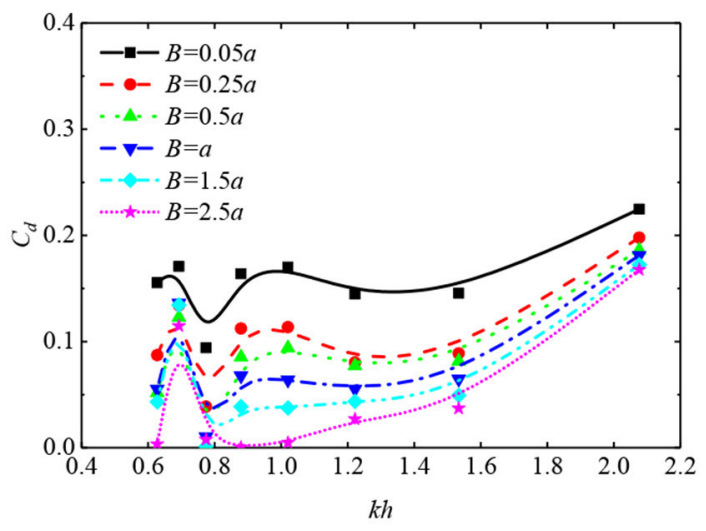

Energy dissipation coefficient

FIGURE 7 | Hydrodynamic coefficients of oscillating water column (OWC) devices with six widths of the rear box under regular waves of $H_{i}=0.05 \mathrm{~m}$. (A) Energy conversion efficiency. (B) Reflection coefficient. (C) Transmission coefficient. (D) Energy dissipation coefficient.

the draft, namely from $d_{2}=7.5 d_{1}$ to $10 d_{1}$, the efficiency improvement is not obvious, as seen in Figure 8A. Similarly, considering $\xi=0.6$ as high efficiency, the highly effective bandwidth for the deepest draft case $d_{2}=10 d_{1}$ is in the range of $1.07 \leq k h \leq 2.08$, while the bandwidth for $d_{2}=d_{1}$ is very narrow, only near $k h=2.08$. This is mainly because a larger draft prevents more waves from transmitting to the rear fields beneath the structure, thus being able to be trapped in the chamber and extracted, which is consistent with the trend of transmission coefficient $C_{t}$ in Figure 8C.

Figure 8B plots the fitting curve of the reflection coefficient $C_{r}$ changing with $k h$. With the draft of rear box increasing, the long wave is reflected more, but the short wave reflection decreases slightly. Combined with the results of the energy dissipation coefficient $C_{d}$ in Figure 8D, it may be that the turbulence and nonlinear effect are quite violent at this time, so the energy of short wave is dissipated more. Sincerely, the large submerged depth of rear box can improve the efficiency, but when the draft increases further from $d_{2}=7.5 d_{1}$ to $10 d_{1}$, the energy conversion efficiency increase is not significant, especially in the high wave frequency region. Taking the difficulty and cost of actual engineering construction into account, $d_{2}=7.5 d_{1}$ of rear box draft is recommended in this study.

\subsection{Effects of the Incident Wave Height}

In this subsection, effects of the incident wave height on hydrodynamic performance of the coupling system were examined numerically. The draft of rear box is fixed at $d_{2}=7.5 d_{1}$ and the width of rear box is $B=2.5 a$. The hydrodynamic coefficients of the system under three incident wave heights, i.e., $H_{i}=0.02 \mathrm{~m}, 0.03 \mathrm{~m}$ and $0.05 \mathrm{~m}$, are considered and the results are plotted in Figure 9.

Figure 9A illustrates the relationship between energy conversion efficiency $\xi$ and non-dimensional parameter $k h$. 
A

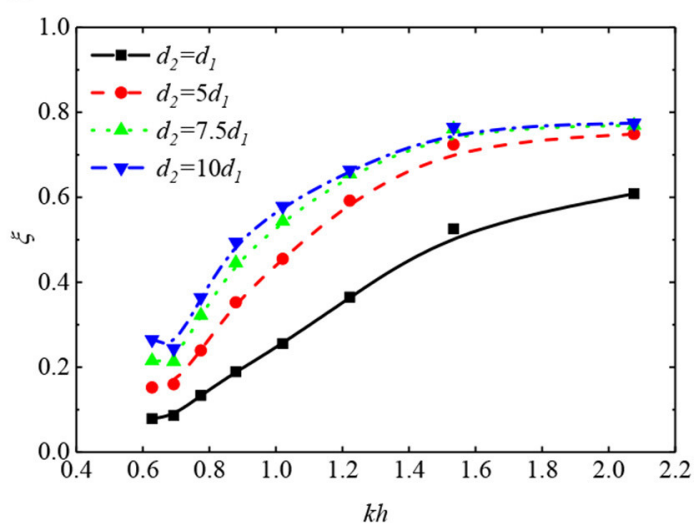

Energy conversion efficiency

C

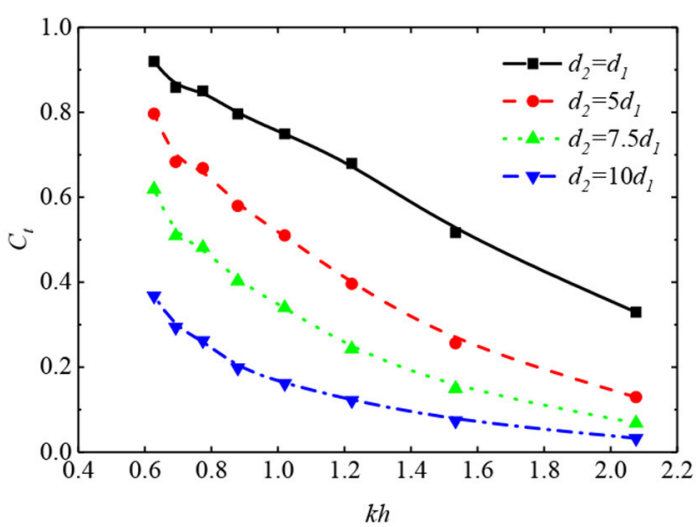

Transmission coefficient
B

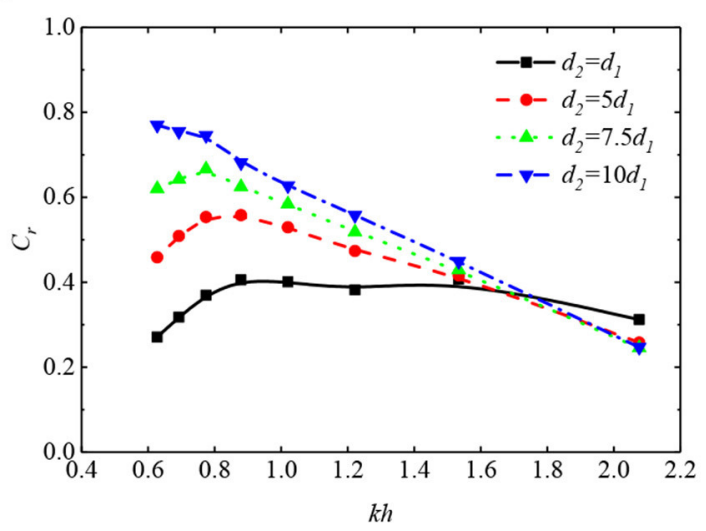

Reflection coefficient

D

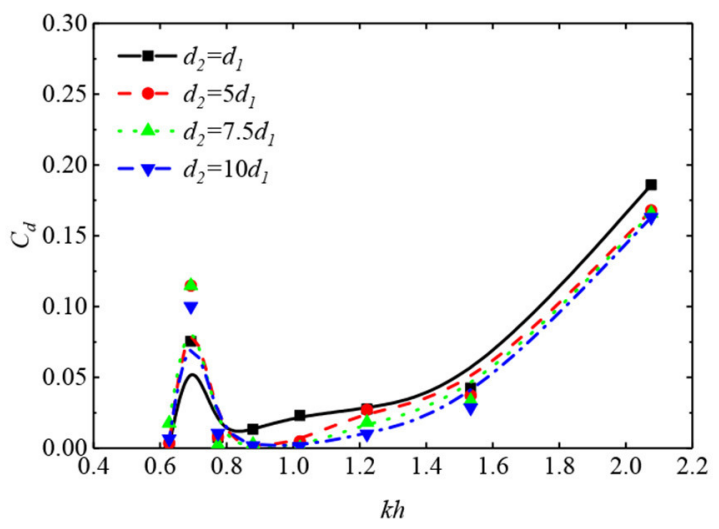

Energy dissipation coefficient

FIGURE 8 | Hydrodynamic coefficients of oscillating water column (OWC) devices with four drafts of the rear box in regular waves of $H_{i}=0.05 \mathrm{~m}$. (A) Energy conversion efficiency. (B) Reflection coefficient. (C) Transmission coefficient. (D) Energy dissipation coefficient.

There exists a turning point at $k h=1.8$. More specifically speaking, for waves whose frequency is less than the turning frequency, steeper waves can enhance the absorption efficiency of the OWC device. However, if larger than this frequency, the efficiency decreases duo to the wave nonlinearity and energy dissipation. Figure 9B shows the effect of wave height on reflection coefficient. It reveals that the steeper the wave height, the less the reflection coefficient over the wave frequency range considered. As expected, the effects of the wave height on the transmission property is neglectable as shown in Figure 9C. The energy dissipation coefficient $C_{d}$ affected by wave height is shown in Figure 9D. Remarkably, within the high frequency domain (i.e., $k h>1.8$ ), the dissipation of wave energy increases obviously with the increase of incident wave height. This is due to that more wave energy transfers from the fundamental frequency to higher-harmonic components, which increases the turbulent intensity. This also explains that the extracted part of the wave energy is reduced when $k h$ is 2.08 , as shown in Figure 9A. However, it should be noted that the low wave energy conversion efficiency does not mean that the total energy extracted from ocean waves is less as well.

\subsection{Comparison Between a Breakwater With OWC Device and a Conventional Box-Type Breakwater}

To further demonstrate the superiority of the proposed OWC device coupled with a box-type breakwater compared to the conventional box-type breakwater, this section tests the hydrodynamic performances of the OWC device and the traditional breakwater under the same wave conditions. The dimension of the present coupling system is the optimal one, i.e., $B=2.5 a, d_{2}=7.5 d_{1}$, and the width of traditional breakwater is equal to the total width of the coupling system, namely $B+a$. 
A

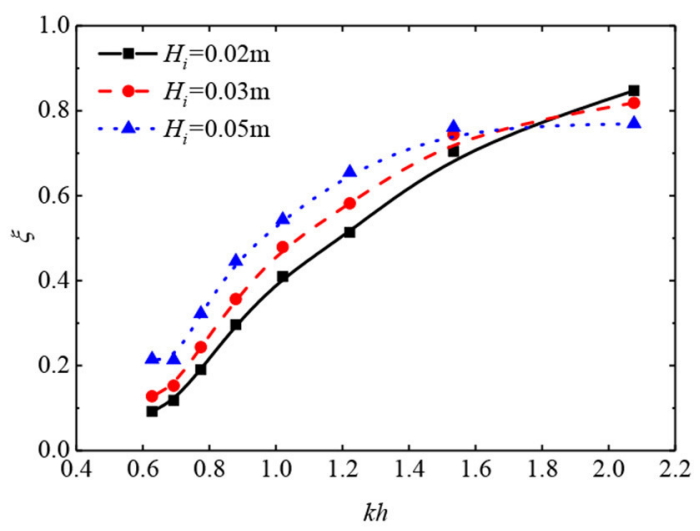

Energy conversion efficiency

C

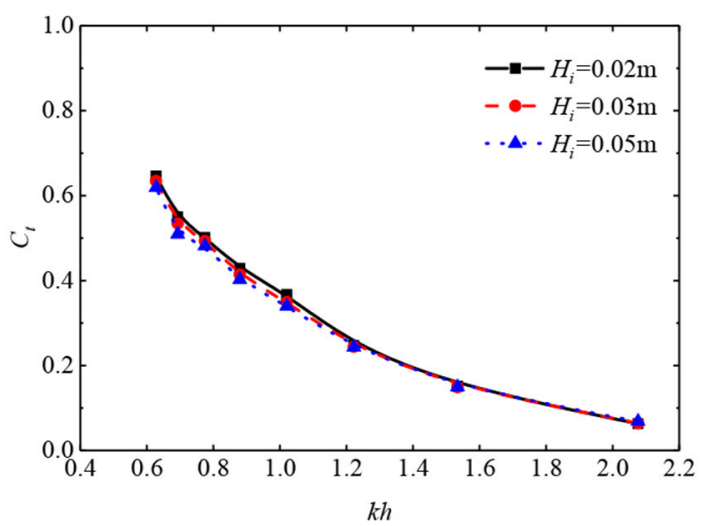

Transmission coefficient
B

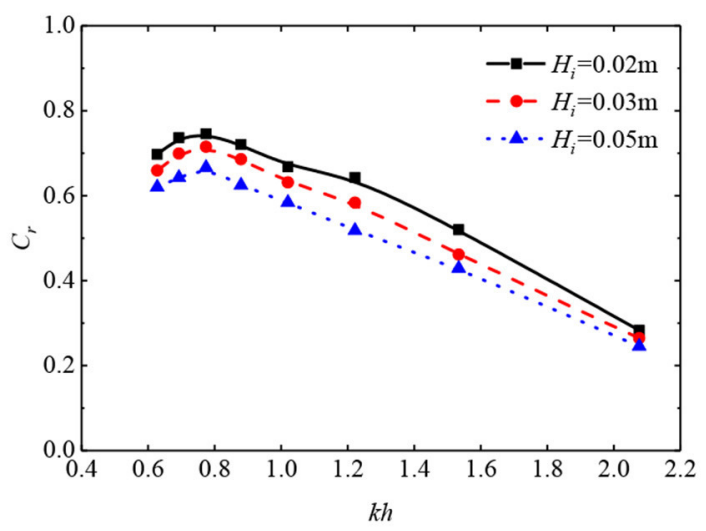

Reflection coefficient

D

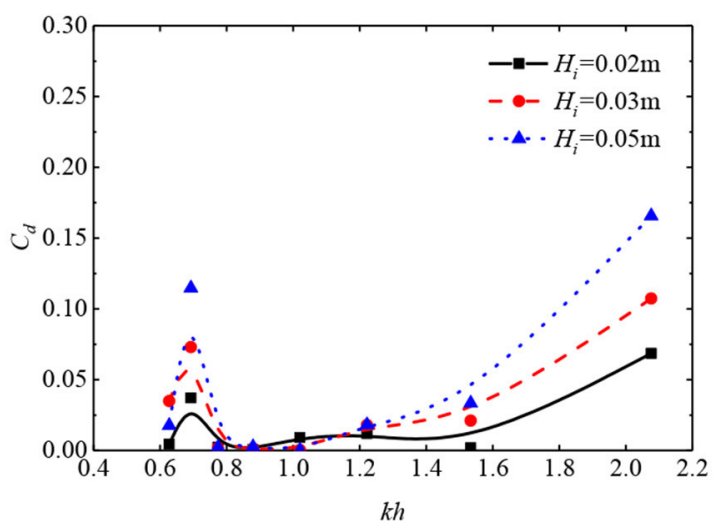

Energy dissipation coefficient

FIGURE 9 | Hydrodynamic coefficients of OWC devices with three incident wave heights for $d_{2}=7.5 d_{1}, B=2.5 a$. (A) Energy conversion efficiency. (B) Reflection coefficient. (C) Transmission coefficient. (D) Energy dissipation coefficient.

The variations of reflection/transmission coefficients and energy dissipation ratio against $k h$ are shown in Figure 10, respectively.

Figure 10A shows that the OWC device can significantly reduce the reflection coefficient of the breakwater structure, and the maximum reduction is up to the $75 \%$. Figure $\mathbf{1 0 B}$ indicates that the transmission coefficient of the OWC device rises very slightly. Although part of the solid structure of the traditional box-type breakwater is transformed into an air chamber in present OWC device, it can basically maintain the resistance performance. More importantly, it also weakens the wave reflection greatly in front of the device and provides more stable sea conditions for ship navigation.

The fitting curves using B-Spline algorithm for the energy dissipation ratio of the two breakwaters are plotted in Figure 10C. It can be found that the dissipation coefficient of the present device is larger than that of traditional breakwater at $k h=0.69$ and $k h=2.08$. As explained in sections 5.1-5.3, the increment of energy loss of present breakwater may be caused by the formation and shedding of vortices in the proximity of the convex corners of structure, as well as the violent turbulence intensity and nonlinear effect.

\section{SUMMARY AND CONCLUSIONS}

In this study, the hydrodynamic performance of an integrated OWC-breakwater system is investigated experimentally and numerically. In addition to the geometric dimensions, including the width $(B)$ and draft $\left(d_{2}\right)$ of rear box, the influence of incoming wave height $\left(H_{i}\right)$ is examined as well. The hydrodynamic parameters, such as wave energy extraction efficiency $(\xi)$, 


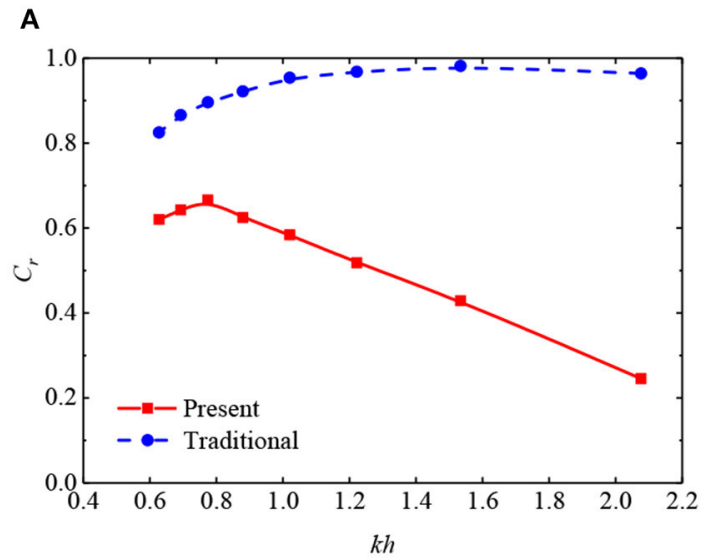

Reflection coefficient
B

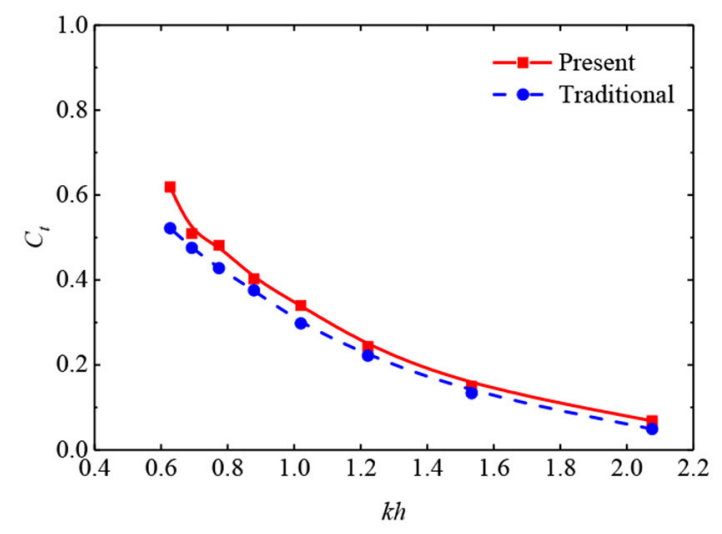

Transmission coefficient

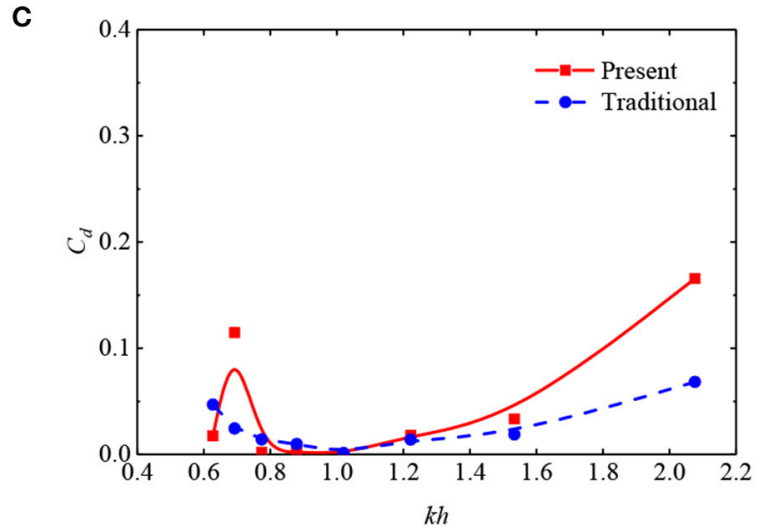

Energy dissipation coefficient

FIGURE 10 | Hydrodynamic coefficients of oscillating water column (OWC) device and traditional breakwater with three incident wave heights for $d_{2}=7.5 d_{1}$, $B=2.5 a$. (A) Reflection coefficient. (B) Transmission coefficient. (C) Energy dissipation coefficient.

reflection coefficient $\left(C_{r}\right)$, transmission coefficient $\left(C_{t}\right)$, and energy dissipation coefficient $\left(C_{d}\right)$ are illustrated. Moreover, the superiority of the coupling system, compared to traditional boxtype breakwater, is discussed. The following conclusions can be drawn:

1. Compared with the traditional OWC device with thin side wall, the hydrodynamic performance of the OWC device, including wave energy conversion efficiency and wave dissipation performance, can be significantly improved by equipping the rear wall with a box structure of considerable width.

2. The draft of rear box has a profound influence on both the energy contraction and dissipation. Taking the wave energy conversion efficiency and the practical project's cost into full consideration, it is sufficient to adopt the immersion depth of rear box with $d_{2}=7.5 d_{1}$.
3. Increasing the incident wave height can improve the wave energy conversion ability except for the short-wave regimes, where more wave energy is dissipated in the front-lip of OWC device due to flow separation and vortex shedding. However, the influence on the wave-blocking ability can be omitted.

4. Compared to the traditional box-type breakwater, the OWCbreakwater coupling system can extract wave energy and simultaneously decrease reflected waves, which is beneficial for ship navigation. In addition, the nearly equivalent waveblocking performance can be obtained.

Based on the well-validated numerical model of water wave interaction with the OWC device, it is proved that the OWCbreakwater coupling system is beneficial for both wave utilization and navigation safety. However, it should be mentioned that the present system is fixed on water surface. The motion response has 
significant influence on the hydrodynamic performance, which needs to be investigated in future.

\section{DATA AVAILABILITY STATEMENT}

The raw data supporting the conclusions of this article will be made available by the authors, without undue reservation.

\section{AUTHOR CONTRIBUTIONS}

ZD proposed the idea and gave the essential suggestions on manuscript writing. PW performed the numerical simulations, conducted experiment tests, and collected and organized the results. PC conducted the experiment tests and gave valuable

\section{REFERENCES}

Ashlin, S. J., Sundar, V., and Sannasiraj, S. A. (2016). Effects of bottom profile of an oscillating water column device on its hydrodynamic characteristics. Renew. Energy 96, 341-353. doi: 10.1016/j.renene.2016.04.091

Count, B. M., and Evans, D. V. (1984). The influence of projecting sidewalls on the hydrodynamic performance of wave-energy devices. J. Fluid Mech. 145, 361-376. doi: 10.1017/S0022112084002962

Deng, Z., Huang, Z., and Law, A. W. K. (2013). Wave power extraction by an axisymmetric oscillating-water-column converter supported by a coaxial tube-sector-shaped structure. Appl. Ocean Res. 42, 114-123. doi: 10.1016/j.apor.2013.05.006

Deng, Z., Huang, Z., and Law, A. W. K. (2014). Wave power extraction from a bottom-mounted oscillating water column converter with a V-shaped channel. Proc. R. Soc. A Math. Phys. Eng. Sci. 470, 20140074. doi: 10.1098/rspa.2014.0074

Deng, Z., Wang, C., Wang, P., Higuera, P., and Wang, R. (2019). Hydrodynamic performance of an offshore-stationary owc device with a horizontal bottom plate: experimental and numerical study. Energy 187:115941. doi: 10.1016/j.energy.2019.115941

Deng, Z., Wang, C., Yao, Y., and Higuera, P. (2020). Numerical simulation of an oscillating water column device installed over a submerged breakwater. J. Marine Sci. Technol. 25, 258-271. doi: 10.1007/s00773-019-00645-0

Deshpande, S. S., Anumolu, L., and Trujillo, M. F. (2012). Evaluating the performance of the two-phase flow solver interFoam. Comput. Sci. Discov. 5:014016. doi: 10.1088/1749-4699/5/1/014016

Devolder, B., Troch, P., and Rauwoens, P. (2018). Performance of a buoyancymodified $\mathrm{k}-\omega$ and $\mathrm{k}-\omega$ SST turbulence model for simulating wave breaking under regular waves using OpenFOAM ${ }^{\circledR}$. Coast. Eng. 138, 49-65. doi: 10.1016/j.coastaleng.2018.04.011

Evans, D. V. (1976). A theory for wave-power absorption by oscillating bodies. J. Fluid Mech. 77, 1-25. doi: 10.1017/S0022112076001109

Evans, D. V. (1982). Wave-power absorption by systems of oscillating surface pressure distributions. J. Fluid Mech. 114, 481-499. doi: $10.1017 /$ S0022112082000263

Falcão, A. F. d. O. (2010). Wave energy utilization: a review of the technologies. Renew. Sustain. Energy Rev. 14, 899-918. doi: 10.1016/j.rser.2009.11.003

Goda, Y., and Suzuki, Y. (1976). Estimation of incident and reflected waves in random wave experiments. Coast. Eng. Proc. 1, 47. doi: 10.9753/icce.v15.47

He, F., Huang, Z., and Law, A. W. K. (2013). An experimental study of a floating breakwater with asymmetric pneumatic chambers for wave energy extraction. Appl. Energy 106, 222-231. doi: 10.1016/j.apenergy.2013.01.013

Heath, T. V. (2012). A review of oscillating water columns. Philos. Trans. R. Soc. A Math. Phys. Eng. Sci. 370, 235-245. doi: 10.1098/rsta.2011.0164

Hirt, C. W., and Nichols, B. D. (1981). Volume of fluid (VOF) method for the dynamics of free boundaries. J. Comput. Phys. 39, 201-225. doi: 10.1016/0021-9991(81)90145-5 discussions of this study. All authors contributed to the article and approved the submitted version.

\section{FUNDING}

We would like to acknowledge the financial support of the National Natural Science Foundation of China (Grants nos. 11802313, 11702244) and the Strategic Priority Research Program of the National Key R\&D Program of China (Grants nos. 2018YFC1505500, 2018YFC1505504).

\section{ACKNOWLEDGMENTS}

The authors thank Prof. Haijiang Liu from Zhejiang University for valuable discussions.

Howe, D., and Nader, J. R. (2017). OWC WEC integrated within a breakwater versus isolated: Experimental and numerical theoretical study. Int. J. Mar. Energy 20, 165-182. doi: 10.1016/j.ijome.2017.07.008

Hu, Z., Greaves, D., and Raby, A. (2016). Numerical wave tank study of extreme waves and wave-structure interaction using OpenFOAM. Ocean Eng. 126:329342. doi: 10.1016/j.oceaneng.2016.09.017

Jacobsen, N. G., Fuhrman, D. R., and Fredsøe, J. (2012). A wave generation toolbox for the open-source CFD library: OpenFOAM. Int. J. Numer. Methods Fluids 70, 1073-1088. doi: 10.1002/fld.2726

Lovas, S., Mei, C. C., and Liu, Y. (2010). Oscillating water column at a coastal corner for wave power extraction. Appl. Ocean Res. 32, 267-283. doi: 10.1016/j.apor.2010.06.004

Martins-Rivas, H., and Mei, C. C. (2009a). Wave power extraction from an oscillating water column along a straight coast. Ocean Eng. 36, 426-433. doi: 10.1016/j.oceaneng.2009.01.009

Martins-Rivas, H., and Mei, C. C. (2009b). Wave power extraction from an oscillating water column at the tip of a breakwater. J. Fluid Mech. 626, 395-414. doi: 10.1017/S0022112009005990

Mustapa, M. A., Yaakob, O. B., Ahmed, Y. M., Rheem, C. K., Koh, K. K., and Adnan, F. A. (2017). Wave energy device and breakwater integration: A review. Renew. Sustain. Energy Rev. 77, 43-58. doi: 10.1016/j.rser.2017. 03.110

Ning, D., Guo, B., Wang, R., Vyzikas, T., and Greaves, D. (2020a). Geometrical investigation of a U-shaped oscillating water column wave energy device. Appl. Ocean Res. 97:102105. doi: 10.1016/j.apor.2020. 102105

Ning, D., Ke, S., Mayon, R., and Zhang, C. (2019). Numerical investigation on hydrodynamic performance of an OWC wave energy device in the stepped bottom. Front. Energy Res. 7:152. doi: 10.3389/fenrg.2019. 00152

Ning, D., Zhou, Y., Mayon, R., and Johanning, L. (2020b). Experimental investigation on the hydrodynamic performance of a cylindrical dualchamber oscillating water column device. Appl. Energy 260:114252. doi: 10.1016/j.apenergy.2019.114252

Qiu, S., Liu, K., Wang, D., Ye, J., and Liang, F. (2019). A comprehensive review of ocean wave energy research and development in China. Renew. Sustain. Energy Rev. 113:109271. doi: 10.1016/j.rser.2019.109271

Rusche, H. (2003). Computational Fluid Dynamics of Dispersed Two-phase Flows at High Phase Fractions. Ph.D. thesis, Imperial College London (University of London).

Vyzikas, T., Deshoulières, S., Barton, M., Giroux, O., Greaves, D., and Simmonds, D. (2017). Experimental investigation of different geometries of fixed oscillating water column devices. Renew. Energy 104, 248-258. doi: 10.1016/j.renene.2016.11.061

Weller, H. (2002). Derivation, Modelling and Solution of the Conditionally Averaged Two-phase Flow Equations. Nabla Ltd., No Technical Report TR/HGW 2. 
Xu, C., Huang, Z., and Deng, Z. (2016). Experimental and theoretical study of a cylindrical oscillating water column device with a quadratic power take-off model. Appl. Ocean Res. 57, 19-29. doi: 10.1016/j.apor.2016. 02.003

You, Y., Li, W., Liu, W., Li, X., and Wu, F. (2010). Development status and perspective of marine energy conversion systems. Autom. Electr. Power Syst. $34,1-12$.

Zhao, X., Ning, D., Zou, Q., Qiao, D., and Cai, S. (2019). Hybrid floating breakwater-wec system: a review. Ocean Eng. 186, 106126-106126. doi: 10.1016/j.oceaneng.2019.106126

Zheng, S., Zhang, Y., and Iglesias, G. (2019). Coast/breakwaterintegrated OWC: a theoretical model. Mar. Struct. 66, 121-135. doi: 10.1016/j.marstruc.2019.04.001

Zheng, S., Zhu, G., Simmonds, D., Greaves, D., and Iglesias, G. (2020). Wave power extraction from a tubular structure integrated oscillating water column. Renew. Energy 150, 342-355. doi: 10.1016/j.renene.2020. 01.008

Conflict of Interest: The authors declare that the research was conducted in the absence of any commercial or financial relationships that could be construed as a potential conflict of interest.

Copyright $\odot 2021$ Deng, Wang and Cheng. This is an open-access article distributed under the terms of the Creative Commons Attribution License (CC BY). The use, distribution or reproduction in other forums is permitted, provided the original author(s) and the copyright owner(s) are credited and that the original publication in this journal is cited, in accordance with accepted academic practice. No use, distribution or reproduction is permitted which does not comply with these terms. 